Pathologe 2010 · [Suppl 2] 31:121-125 DOI 10.1007/s00292-010-1345-7

Online publiziert: 28. August 2010

(c) Springer-Verlag 2010

\title{
S. Perner
}

Institut für Pathologie, Comprehensive Cancer Center, Universitätsklinikum Tübingen

"Dangerous liaisons" im Prostatakarzinom

\section{Klinische und biologische Bedeutung rekurrenter Genfusionen}

tischer Ansatz zur Entschlüsselung onkogener Profile aus Expressionsdatensätzen und die daraus resultierende Identifikation überexprimierter Gene, die häufig mit Genrearrangements in anderen Tumoren einhergehen. Bei diesen Untersuchungen zeigten zwei Gene, nämlich $E R G$ und ETV1, konstant hohe Expressionswerte bei Prostatakarzinom-Microarrays. Diese beiden Gene sind Teil der Familie der ETS-Transkriptionsfaktoren, in der Mehrheit der Prostatakarzinomfälle überexprimiert und in einem jeweiligen Tumor gegenläufig in ihrem Expressionsprofil. Dies legte den Schluss nahe, dass diese beiden Gene bei der Entstehung des Prostatakarzinoms funktionell redundant sind.

Da die Transkriptionsfaktoren der ETS-Familie bis dato nur in der Translokation von Ewing-Sarkomen, der akuten myeloischen Leukämie (AML) und anderen seltenen Tumoren beobachtet werden konnten, wurde exploriert, ob diese Gene Teil einer Translokation beim Prostatakarzinom sein könnten. Bei der Evaluation des ERG-cDNA-Transkriptes mittels „Exon-Walking“ wurde eine Überexpression der Exone am distalen (3'-) Ende, nicht aber am proximalen (5'-) Ende festgestellt. Durch Sequenzierung der cDNATranskripte wurden Fusionen der nichttranslatierten 5'-Region des TMPRSS2Gens (21q22.3) mit einem der beiden Transkriptionsfaktoren der ETS-Familie, $E R G$ (21q22.2) oder ETV1 (7p21.2), identifiziert, was die Überexpression der ETSGene beim Prostatakarzinom erklärt.

Neben der TMPRSS2-ERG-Fusion, die die häufigste Fusion darstellt, wurden wei- tere, jedoch wesentlich seltenere und bisher wenig charakterisierte Fusionsereignisse beschrieben [5]. Diese weniger häufigen Fusionen demonstrieren die Neigung der ETS-Gene, sich an Rearrangements zu beteiligen.

\section{Genomische Subtypen der TMPRSS2-ERG-Genfusion}

Als Goldstandard für den Fusionsnachweis im Gewebe dient die Fluoreszenzin-situ-Hybridisierung (FISH) als kombinierter zytogenetisch-histomorphologischer Test. Zum Nachweis einer Fusion von TMPRSS2 mit ERG konnten wir keinen direkten Fusions-Assay anwenden, da beide Gene auf dem gleichen Chromosom (21q) nicht ausreichend weit voneinander entfernt liegen (Distanz lediglich etwa 3 Megabasenpaare). Daher mussten wir einen Translokations-Assay etablieren, der das Rearrangement eines der beiden Fusionspartner, z. B. des ERG-Gens, detektiert. Ein positives Translokationssignal wurde in diesem Falle als eine Fusion von ERG mit TMPRSS2 interpretiert. Durch Vergleich der Ergebnisse aus Polymerase-Ketten-Reaktions- (PCR-)Analysen (womit auf Transkriptionsebene die Fusion nachgewiesen werden konnte) und FISH-Analysen (womit die Translokation des ERG-Gens nachgewiesen werden konnte) war dieser Rückschluss zulässig [4].

Das Manuskript entspricht in weiten Teilen der Habilitationsschrift des Autors. 
Allerdings haben sich bei Anwendung des ERG-Translokations-Assays zwei verschiedene Rearrangement-Muster gezeigt. Zum einen, wie erwartet, ein „Break-apart“ der differenziell Fluorochrom-markierten ERG-flankierenden Sonden in zwei Einzelsignale. Zum anderen beobachteten wir überraschenderweise, dass in der Mehrzahl der Fälle die telomerische 5'-ERG-Sonde verloren ging, was nahe legte, dass genomisches Material zwischen ERG und TMPRSS2 einer Deletion unterlag [6]. Daraufhin analysierten wir 30 Prostatakarzinomproben mittels $100 \mathrm{~K}$-Oligonucleotid-SNP-Arrays und fanden heraus, dass sich zwischen TMPRSS 2 und ERG eine homogene Deletion befand.

Durch die Beobachtungen aus der FISH-Analyse und den Ergebnissen aus den SNP-Array-Analysen konnten wir somit zeigen, dass der TMPRSS2-ERGGenfusion zwei unterschiedliche Mechanismen zugrunde liegen: Der häufigere der beiden Mechanismen war eine Fusion durch Deletion genetischen Materials zwischen den beiden Genen. Für den anderen, etwas selteneren Mechanismus wurde bisher eine Translokation oder Insertion favorisiert. Eine biologische Bedeutung der unterschiedlichen Mechanismen konnte allerdings bisher nicht herausgearbeitet werden.

\section{Häufigkeit der Genfusion beim Prostatakarzinom}

Viele unabhängige Studien konnten unsere Beobachtung bestätigten, dass die $T M P R S S_{2}-E R G$-Fusion ein häufiges Ereignis beim Prostatakarzinom ist [5]. Die meisten Studien beschränkten sich auf das dominante Rearrangement der TMPRSS2$E R G$-Fusion. Eine Vielzahl anderer Fusionen, u. a. TMPRSS 2 und weitere 5'-Partner, wurden beschrieben, scheinen aber deutlich weniger häufig vorzukommen und betreffen maximal 1-5\% aller Prostatakarzinome. Für die Prävalenz der TMPRSS 2-ERG-Prostatakarzinome reichen die Werte je nach Kohorte und Untersuchungsmethode von $15-70 \%[5,7]$, wobei Kohorten mit inzidentiell diagnostizierten Prostatakarzinomen signifikant geringere Häufigkeiten aufweisen als PSA-gescreente Kohorten oder Kohorten mit hohem Anteil aggressiv verlaufender Tumoren.

\section{Die TMPRSS2-ERG-Genfusion - ein frühes, klonales und prostata- karzinomspezifisches Ereignis}

Eine unserer ersten Erkenntnisse bei der Untersuchung des Prostatakarzinoms auf die TMPRSS2-ERG-Fusion mittels FISHAssays war, dass diese Alteration ausschließlich in neoplastischen Zellen vorkommt. In einer großen Studie über ein breites Spektrum benigner Prostataläsionen und potenzieller Vorläuferläsionen des Prostatakarzinoms konnten wir zeigen, dass die TMPRSS2-ERG-Fusion nicht in normalen Prostatadrüsen, benigner prostatischer Hyperplasie (BPH) oder entzündlich/atrophischen Veränderungen des Prostataepithels vorkommt [8]. Interessanterweise fanden wir aber die TMPRSS2-ERG-Fusionen in knapp 1/5 der „High-grade-PIN- (prostatischen intraepitheliale Neoplasie-) Läsionen“, die in unmittelbarer Nähe von Karzinomherden mit demselben Fusionsmuster lagen.

Eine von uns groß angelegte Studie und eine unabhängige Studie zur Bedeutung der TMPRSS2-ERG-Genfusion in „Highgrade-PIN-Läsionen “ bestätigte unsere initiale Beobachtung $[9,10]$. Wir werten dies als ein Indiz, dass diese „High-grade-PINs“ eine Untergruppe von Vorstufen des TMPRSS2-ERG-positiven Prostatakarzinoms sind.

Ein signifikanter klinischer Nutzen dieser Erkenntnis ist die Bestimmung des TMPRSS2-ERG-Fusionsstatus bei Prostatastanzbiopsien mit „High-grade-PIN“ und/oder mit kleinen atypischen azinären Drüsenformationen (ASAP) ohne gleichzeitigen Prostatakarzinomnachweis. Aufgrund der Klonalität der TMPRSS2-ERGGenfusion innerhalb eines Fokus und der Tatsache, dass diese Genfusion schon in der potenziellen Vorläuferläsion PIN vorkommt, glauben wir, dass die Genfusion ein frühes Ereignis in der Entwicklung und Progression von Prostatakarzinomen darstellt.

Es ist bekannt, dass das Prostatakarzinom in der großen Mehrzahl der Fälle multifokal auftritt. Erst mit der FISHbasierten Identifizierung der rekurrenten TMPRSS2-ERG-Fusion steht uns ein In-
situ-Klonalitätsmarker zur Verfügung. Wir konnten zeigen, dass die TMPRSS2ERG-Fusion in allen Tumorzellkernen innerhalb eines distinkten Tumorfokus nachweisbar ist [8]. Folglich muss die Genfusion innerhalb dieses Fokus sehr früh stattfinden. Andererseits stellten wir beim Vergleich der Genfusion zwischen verschiedenen Tumorherden innerhalb einer Prostata fest, dass jeder Tumorfokus unabhängig vom anderen eine Genfusion aufweisen kann [11]. In darauf aufbauenden Untersuchungen konnten wir auch zeigen, dass bei multifokalen Prostatakarzinomen immer der Fokus, der durch ein ERG-Rearrangement charakterisiert ist, auch für die Metastasierung in regionäre Lympknoten verantwortich ist [12].

Ein weiterer wesentlicher Punkt in der Charakterisierung des ERG-Rearrangements war die Frage, ob diese Alteration ein prostatakarzinomspezifisches Ereignis darstellt oder auch in anderen epithelialen Tumoren vorkommt. Wir konnten in einer erst kürzlich erschienenen Studie an über 2000 Tumorgewebeproben von über 20 Tumorentitäten herausarbeiten, dass das ERG-Rearrangement nur im Prostatakarzinom und sonst in keinen anderen Karzinomen vorkommt [13]. Interessanterweise konnten wir im Rahmen dieser Untersuchungen auch zeigen, dass die seltene, aber aggressive Erscheinungsform der kleinzelligen Prostatakarzinome wahrscheinlich keine eigene Tumorentität ist, sondern die dedifferenzierte Variante der azinären Prostatakarzinome darstellt [14].

\section{Zusammenhang der TMPRSS2- ERG-Genfusion mit einem aggressiveren klinischen Verlauf}

Unsere Gruppe beobachtete anfangs vermehrt Fälle von TMPRSS2-ERG-Fusion bei fortgeschrittenen Prostatakarzinomen. Daraufhin untersuchten wir, ob ein Zusammenhang zwischen der TMPRSS2$E R G$-Fusion und dem klinischen Outcome in einer populationsbasierten Studie besteht [15]. In der „Watchful-Waiting-Kohorte" inzidenteller Prostatakarzinompatienten aus Örebro (Schweden) wurde in $15 \%$ der Fälle eine TMPRSS2-ERG-Genfusion festgestellt und eine signifikante Assoziation mit prostataspezifischem Tod 
gezeigt. Damit gewannen wir durch ein einzigartiges Studiendesign Hinweise auf die biologischen Auswirkungen des TMPRSS 2-ERG-Prostatakarzinoms unter Ausschluss früher Intervention. Diese Ergebnisse wurden durch eine größer angelegte Studie aus Großbritannien gestützt [16]. Insgesamt zeigten dort Karzinome ohne die TMPRSS2-ERG-Fusion eine 8-Jahres-Überlebensrate von 90\%. Diese Studie unterstützt die These, dass TMPRSS2-ERG eine Bedeutung für ein aggressives biologisches Verhalten hat.

In der Zwischenzeit wurden zahlreiche retrospektive Studien über den Zusammenhang zwischen TMPRSS $2-E R G$ und Wiederanstieg des PSA-Wertes nach radikaler Prostatektomie mit unterschiedlichen Ergebnissen durchgeführt [5]. Es ist allerdings sehr schwierig, Studien mit dem PSA-Rezidiv als Endpunkt mit solchen zu vergleichen, die den krebsspezifischen Tod als Endpunkt werten. Auf der Basis der beiden großen publizierten Studien mit langem Follow-up steht immer noch die These, dass das TMPRSS2-ERGProstatakarzinom unbehandelt einen aggressiveren Verlauf nimmt als ein Fusionsnegativer Tumor. Für die Bewertung chirurgischer oder anderer Interventionen fehlen noch ausreichende Daten. Wir hoffen, diese kontroversen Ergebnisse durch Untersuchung großer prospektiver populationsbasierter Kohorten zu klären.

\section{Weitere kritische Ereignisse im Zusammenhang mit der Genfusion im Prostatakarzinom}

Angesichts der phänotypischen Veränderungen beim TMPRSS2-ERG-FusionsProstatakarzinom und aggressivem klinischem Verlauf könnte man annehmen, dass Fusions-Prostatakarzinome ein individuelles molekulares Profil zeigen. Wir berichteten vor Kurzem von einer molekularen Signatur für das TMPRSS2ERG-Prostatakarzinom, basierend auf den Daten von 455 Prostatakarzinompatienten aus der schwedischen „WatchfulWaiting-Kohorte“ und der „PhysiciansHealth-Study-Kohorte" aus den USA [17]. Eine Genexpressionssignatur für Prostatakarzinome mit der TMPRSS2-ERG-Fusion wurde bestimmt und ergab eine robuste Expressionssignatur bestehend aus

Pathologe 2010 • [Suppl 2] 31:121-125 DOI 10.1007/s00292-010-1345-7

C) Springer-Verlag 2010

\section{S. Perner}

„Dangerous liaisons" im Prostatakarzinom. Klinische und biologische Bedeutung rekurrenter Genfusionen

\section{Zusammenfassung}

Das Prostatakarzinom ist ein häufiges und im klinischen Verlauf sehr heterogenes Malignom des Mannes. Um Fortschritte in der Behandlung von Prostatakarzinompatienten zu erzielen, ist es von höchster Relevanz, die Tumorbiologie dieses Malignoms besser zu verstehen. Damit soll das Risiko für eine Tumorprogression erfasst und Grundlagen für neue rationale Therapeutika geschaffen werden, die das Fortschreiten der Erkrankung verlangsamen oder aufhalten. Die Entdeckung und Charakterisierung von rekurrierenden Rearrangements der ETS-Gene wobei es sich am häufigsten um ein Rearrangement des ERG-Gens handelt - war ein Meilenstein der translationalen Prostatakarzinomforschung. Obwohl schon eine Vielzahl von molekularen Veränderungen im Prosta- takarzinom bekannt sind, sollte ein genaues Verständnis der Genfusionen im Prostatakarzinom dazu beitragen, die klinische und biologische Vielgestaltigkeit des Prostatakarzinoms besser zu verstehen. Die könnte auch Grundlage für eine molekulare Subklassifikation dieses Malignoms sein. Diese Übersichtsarbeit beschreibt den Weg von der Entdeckung der Genfusionen im Prostatakarzinom, über Anwendungsmöglichkeiten in der klinischen Versorgung von Patienten mit Prostatakarzinom bis hin zu daraus folgenden wissenschaftlichen Fragestellungen.

\section{Schlüsselwörter}

Prostatakarzinom - Genfusion . ERG Rearrangement · ETS Gene · Fluoreszenz in-situ Hybridisierung

\section{Dangerous liaisons in prostate cancer. Clinical and biological implications of recurrent gene fusions}

\section{Abstract}

Prostate cancer is a common and clinically heterogeneous disease. Understanding the biology of prostate cancer is necessary to best determinate the risk of disease progression and develop novel therapeutic approaches to prevent or slow down disease progression. The recent discovery and subsequent characterization of recurrent gene rearrangements of ETS genes - most frequently $E R G$ - in the majority of prostate cancers is a milestone in translational prostate cancer research. Although multiple molecular alterations have been detected in prostate cancer, a detailed understanding of gene fusion in prostate cancer should help explain the clinical and biologic diversity in addition to providing a rationale for a molecular sub-classification of the disease. This review describes the path from the identification of common ETS gene rearrangements in prostate cancer to possible applications in the treatment of patients, on to the potential scientific implications arising from their discovery.

\section{Keywords}

Prostate cancer - Gene fusion . ERG rearrangement ETS genes . In situ hybridization, fluorescence 
87 Genen, die den TMPRSS2-ERG-Fusions-Tumor als diskrete molekulare Entität abgrenzte. In-silico-Analysen von Signaltransduktionswegen ergaben, dass die oben genannte Genfusionssignatur mit einer Östrogenrezeptor- (ER-) assoziierten Signalübermittlung einhergeht. Anschließende In-vitro-Tests zeigten, dass eine Stimulation von ER-a zu einer Überexpression des TMPRSS2-ERG-Transkriptes in Fusions-positiven NCI H-66o-Prostatakarzinomzellen führt. Außerdem konnten wir einen ER-Bindungsort innerhalb des TMPRSS 2 -Promotors mittels Chromatin-Immunpräzipitations-Assays nachweisen.

Die Beobachtung der ER-abhängigen Signaltransduktionswege beim TMPRSS2ERG-Prostatakarzinom hat potenzielle klinische Auswirkungen. Die Expression des TMPRSS2-ERG-Fusions-Transkriptes beim kastrationsresistenten Prostatakarzinom legt nahe, dass der TMPRSS2-Promotor auch durch ER- $\alpha$-Stimulation aktiv bleiben kann. Man fand heraus, dass eine erhöhte Expression des ER- $\alpha$ mit Tu- morprogression, Metastasierung und dem kastrationsresistenten Phänotyp einhergeht [18]. Daher könnte die klinische Anwendung von selektiven Östrogenmodulatoren (SERM), welche ER-a-stimulierende Wirkung haben, die Progression TMPRSS2-ERG-abhängiger Prostatakarzinome fördern.

Diese Daten lassen auch einen Mechanismus vermuten, mit dem ER- $\beta$ als Tumorsuppressor wirken könnte, da es die TMPRSS2-ERG-Expression negativ reguliert [19]. In Experimenten an Zelllinien fanden wir heraus, dass die Aktivierung von ER- $\beta$ die Expression von TMPRSS2$E R G$ tatsächlich vermindert. Diese Ergebnisse verdeutlichen die Notwendigkeit, ER- $\beta$-spezifische Agonisten in der Behandlung des Prostatakarzinoms zu testen und die therapeutische Verwendung von Arzneimitteln mit ER- $\alpha$-agonistischer Aktivität mit großer Aufmerksamkeit zu beobachten und zu hinterfragen.

Als vielleicht wichtigsten Punkt lassen unsere Ergebnisse einen Mechanismus vermuten, mit dem Prostatakarzi- nome eine Androgenunabhängigkeit entwickeln können, obwohl sie initial androgenabhängig waren. Insbesondere wird das TMPRSS2-ERG-Onkogen durch Östrogenrezeptoren reguliert, wobei ERa-Agonisten (z. B. endogene Östrogene) die Onkogenexpression stimulieren können. Diese Experimente legen nahe, dass eine pharmakologische Inhibition der TMPRSS2-ERG-Expression mittels ER-aAntagonisten mit ER- $\beta$-agonistischer Aktivität ein viel versprechender neuer Therapieansatz für das Prostatakarzinom sein kann.

\section{Genfusionen in anderen häufigen epithelialen Tumoren}

Mit der Entdeckung rekurrenter Genfusionen im Prostatakarzinom als einem der häufigsten Tumoren des Mannes hat sich ein Paradigmenwechsel vollzogen. Mit dieser Entdeckung wurde postuliert, dass auch andere häufige epitheliale Tumoren ähnliche organspezifische Rearrangements aufweisen müssten. Schon im Jahr 
2007 haben zwei unabhängige Gruppen eine rekurrente Genfusion im nichtkleinzelligen Lungenkarzinom aufdecken können $[20,21]$. Dabei handelt es sich um die Fusion zwischen der analplastischen Lymphomkinase $(A L K)$ und $E M L 4$, einem noch sehr unbekannten Gen. ALK kodiert eine Tyrosinkinase, die schon in anaplastischen Lymphomen als Teil einer Fusion beschrieben ist. Diese Fusion wurde in knapp 7\% einer Gruppe von japanischen Lungenkarzinompatienten nachgewiesen [20]. Durch eine Studie konnten wir den ersten In-situ-Nachweis erbringen, dass die beiden Gene EML4 und ALK tatsächlich rearrangiert sind [22].

Da schon bald nach der TMPRSS2ETS-Genfusion im Prostatakarzinom eine rekurrente Genfusion auch in einem anderen sehr häufigen Tumor entdeckt wurde, gehen wir davon aus, dass sich auch im nichtkleinzelligen Lungenkarzinom und anderen häufigen epithelialen Tumoren wie dem Mamma- und Kolonkarzinom weitere pathognomonische Genfusionen mit klinischer Bedeutung finden lassen.

\section{Fazit für die Praxis}

Die kürzliche Entdeckung rekurrenter Genfusionen im Prostatakarzinom gilt als wissenschaftlicher Meilenstein in der Genetik solider Tumoren. Damit hat sich ein neues Forschungsfeld aufgetan, das mehr neue Fragen aufwirft, als es bisher beantworten kann. Die Herausforderung wird sein, u. a. folgende Fragen zu beantworten:

- Kommen charakteristische Genfusionen in allen humanen Malignomen vor?

- Warum gibt es Genfusionen? DNADoppelstrangbrüche sind die Basis für alle Genrearrangements und resultieren aus hochenergetischer Strahlung oder Karzinogenen. Wie beim Prostatakarzinom konnte für die meisten Genrearrangements keine spezifische Ursache identifiziert werden.

- Wann entsteht eine Genfusion? Für viele Tumoren, die durch eine Genfusion charakterisiert sind, gibt es keine präneoplastischen Zellen, die man auf die Genfusion untersuchen kann.
- Sind Genfusionen als alleiniges Ereignis für eine neoplastische Transformation ausreichend?

- Können Genfusionen als zuverlässige prognostische oder prädiktive Marker und als Grundlage einer rationalen Therapie herangezogen werden?

\section{Korrespondenzadresse \\ PD Dr. S. Perner}

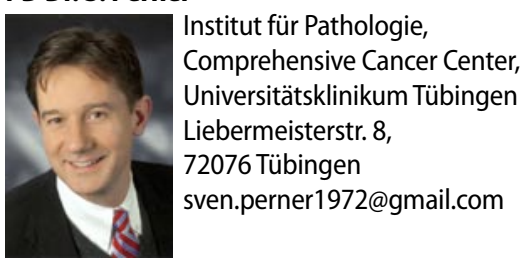

Danksagung. Mein besonderer Dank gilt meinen wissenschaftlichen und klinisch-pathologischen Lehrern Professor Dr. P. Möller, Professor Dr. M. Rubin und Professor F. Fend, deren Mitarbeitern, den Mitarbeitern meiner Arbeitsgruppe und allen Kooperationspartnern. Der Deutschen Forschungsgemeinschaft (DFG) danke ich für die Förderung meiner Arbeiten im Rahmen von Forschungsstipendien und der Möglichkeit des Aufbaus einer eigenen Arbeitsgruppe im Rahmen eines Emmy-Noether-Nachwuchsgruppenprogramms.

Interessenkonflikt. Der korrespondierende Autor weist auf folgende Beziehung hin: PD Dr. S. Perner ist Co-Patenthalter für den diagnostischen Einsatz der ETS-Gen-Rearrangements. Das Patent wurde von GenProbe Inc. lizensiert.

\section{Literatur}

1. Mitelman F (2000) Recurrent chromosome aberrations in cancer. Mutat Res 462(2-3):247-253

2. Mitelman F, Johansson B, Mertens F (2004) Fusion genes and rearranged genes as a linear function of chromosome aberrations in cancer. Nat Genet 36(4):331-334

3. Mitelman F, Johansson B, Mertens F (2007) The impact of translocations and gene fusions on cancer causation. Nat Rev Cancer 7(4):233-245

4. Tomlins SA, Rhodes DR, Perner S et al (2005) Recurrent fusion of TMPRSS2 and ETS transcription factor genes in prostate cancer. Science 310(5748):644-648

5. Tomlins SA, Bjartell A, Chinnaiyan AM et al (2009) ETS gene fusions in prostate cancer: from discovery to daily clinical practice. Eur Urol 56(2):275-286

6. Perner S, Demichelis F, Beroukhim R et al (2006) TMPRSS2:ERG fusion-associated deletions provide insight into the heterogeneity of prostate cancer. Cancer Res 66(17):8337-8341

7. Braun M, Scheble VJ, Scharf G et al (o J) Relevance of cohort design for studying the prevalence of the ERG rearrangement in prostate cancer. (manuscript in review)

8. Perner S, Mosquera JM, Demichelis F et al (2007) TMPRSS2-ERG fusion prostate cancer: an early molecular event associated with invasion. Am J Surg Pathol 31(6):882-888
9. Mosquera JM, Perner S, Genega EM et al (2008) Characterization of TMPRSS2-ERG fusion high-grade prostatic intraepithelial neoplasia and potential clinical implications. Clin Cancer Res 14(11):33803385

10. Cerveira N, Ribeiro FR, Peixoto A et al (2006) TMPRSS2-ERG gene fusion causing ERG overexpression precedes chromosome copy number changes in prostate carcinomas and paired HGPIN lesions. Neoplasia 8(10):826-832

11. Barry M, Perner S, Demichelis F et al (2007) TMPRSS2-ERG fusion heterogeneity in multifocal prostate cancer: clinical and biologic implications. Urology 70(4):630-633

12. Perner S, Svensson MA, Hossain RR et al (2009) ERG rearrangement metastasis patterns in locally advanced prostate cancer. Urology 75(4)762-767

13. Scheble VJ, Braun M, Wilbertz T et al (2010) ERG rearrangement is specific to prostate cancer and does not occur in any other common tumor. Mod Pathol

14. Scheble VJ, Braun M, Wilbertz T et al (2010) ERG rearrangement in small cell prostatic and lung cancer. Histopathology 56(7):937-943

15. Demichelis F, Fall K, Perner S et al (2007) TMPRSS2: ERG gene fusion associated with lethal prostate cancer in a watchful waiting cohort. Oncogene 26(31):4596-4599

16. Attard G, Clark J, Ambroisine L et al (2008) Duplication of the fusion of TMPRSS2 to ERG sequences identifies fatal human prostate cancer. Oncogene 27(3):253-263

17. Setlur SR, Mertz KD, Hoshida Y et al (2008) Estrogen-dependent signaling in a molecularly distinct subclass of aggressive prostate cancer. J Natl Cancer Inst 100(11):815-825

18. Bonkhoff H, Fixemer T, Hunsicker l et al (2001) Progesterone receptor expression in human prostate cancer: correlation with tumor progression. Prostate 48(4):285-291

19. Cheng J, Lee EJ, Madison LD et al (2004) Expression of estrogen receptor beta in prostate carcinoma cells inhibits invasion and proliferation and triggers apoptosis. FEBS Lett 566(1-3):169-172

20. Soda M, Choi YL, Enomoto M et al (2007) Identification of the transforming EML4-ALK fusion gene in non-small-cell lung cancer. Nature 448(7153):561-566

21. Rikova K, Guo A, Zeng Q et al (2007) Global survey of phosphotyrosine signaling identifies oncogenic kinases in lung cancer. Cell 131(6):1190-1203

22. Perner S, Wagner PL, Demichelis F et al (2008) EML4-ALK fusion lung cancer: a rare acquired event. Neoplasia 10(3):298-302 